\title{
APRESENTAÇÃO DO NÚMERO
}

Isabel Soares ${ }^{1}$

\section{Contributos da investigação sobre a vinculação em Portugal}

Este número temático reúne um conjunto de investigações sobre a vinculação, conduzidas em Portugal, no âmbito de distintos grupos ${ }^{2}$, incidindo em temáticas distintas relativas à infância, adolescência e idade adulta, orientadas para problemáticas e contextos específicos, tais como a maternidade na adolescência, a adopção, as relações de amor, de amizade e de trabalho, recorrendo a métodos distintos de avaliação da vinculação, incluindo entrevistas, questionários, observações da interacção diádica com base em escalas e em $Q$-set.

No seu conjunto, este corpo de investigação espelha a multiplicidade dos temas e a variedade de linhas de investigação que hoje se cruzam no território científico da investigação da vinculação. Na esteira da investigação internacional, os diversos grupos de investigação da vinculação em Portugal procuram examinar e responder a questões e desafios da actualidade, recorrem a métodos de avaliação correntes neste domínio científico e oferecem contributos no plano da metodologia, por exemplo, questionando e afinando medidas existentes.

Neste âmbito, o primeiro estudo apresentado, da autoria de Jongenelen e colaboradores, procura examinar a questão da transmissão intergeracional da vinculação num grupo de mães adolescentes e seus bebés, cruzando duas medidas centrais na investigação da vinculação: a Adult Attachment Interview (George, Kaplan \& Main, 1985) e a Situação Estranha (Ainsworth \& Wittig, 1969; Ainsworth \& Bell, 1970; Ainsworth, Blehar, Waters \& Wall, 1978). Este estudo com uma amostra de risco revela uma

\footnotetext{
${ }^{1}$ Professora catedrática do Departamento de Psicologia, Instituto de Educação e Psicologia, Universidade do Minho. Coordenadora do Grupo de Estudos sobre a Vinculação (GEV) na Universidade do Minho. Correspondência sobre este número temático poderá ser enviada para o e-mail isoares@iep.uminho.pt.

${ }^{2}$ Grupos de investigação associados às universidades do Minho, Porto, Coimbra e Lisboa e ao Instituto Superior de Psicologia Aplicada.
} 
distribuição dos padrões de vinculação nos bebés muito próxima da encontrada em amostras consideradas "normativas", ao nível nacional (Soares, 1996; Soares et al., 1995) e internacional (van IJzendoorn \& Kroonenberg, 1988). Por outro lado, a preponderância de mães adolescentes com uma organização de vinculação insegura aproxima-se também da distribuição obtida em outras pesquisas com mães de nível socioeconómico baixo (van IJzendoorn \& Bakermans-Kranenburg, 1996). Contudo, em contraste com resultados encontrados em outros estudos, (por exemplo, Fonagy et al., 1991; Benoit \& Parker 1994), esta amostra portuguesa não revelou uma concordância significativa entre a organização da vinculação da mãe e do seu bebé. A questão da transmissão intergeracional da vinculação continua em aberto, para a qual este artigo procura contribuir.

O segundo artigo de Veríssimo e Salvaterra centra-se temática da adopção e procura examinar duas questões relevantes no processo de construção da relação de vinculação - a idade da criança, na altura da adopção, tem influência na qualidade da vinculação estabelecida com a sua nova família? Há relação entre o modelo interno dinâmico da mãe e o comportamento de base segura do seu filho(a) adoptado? Para analisar estas questões, foram utilizados o Attachment Behaviour Q-Set e as Narrativas de Representação da Vinculação em Adultos. Os resultados encontrados trazem para primeiro plano a discussão da noção de período crítico para o estabelecimento da vinculação e, por outro lado, salientam a importância de uma figura estável e sensível para a promoção e desenvolvimento da relação de vinculação, apoiando a relevância da sensibilidade materna, conceito-chave de Ainsworth, na qualidade deste novo contexto relacional construído pela adopção. Além disso, este novo território relacional remete-nos, também, para a questão da transgeracionalidade da vinculação já abordada no artigo anterior de Jongenelen e colaboradores, no contexto da maternidade na adolescência - no sentido em que que o modelo interno da mãe poderá constituir um factor mediador da qualidade da prestação dos cuidados e da interacção que esta estabelece com o seu filho(a), e que se reflecte na qualidade de vinculação das crianças.

$\mathrm{O}$ artigo de Lima e colaboradores avança para um outro território relacional - as relações íntimas na idade adulta - procurando cruzar a abordagem centrada no discurso e na representação do indivíduo, a partir da Intimate Relationship Interview (Lima, Vieira, Soares \& Collins, 2005), com o foco na interacção do casal e nos seus comportamentos em situações problemáticas, com base na Couple Interaction Task (Collins, Hennighausen, Madsen \& Roisman, 1998). Os resultados preliminares apontam para a associação entre estes dois níveis de análise e para a pertinência da combinação de múltiplos métodos na avaliação da vinculação no contexto das relações íntimas na idade adulta. 
Figueiredo e colaboradores analisam a qualidade da vinculação e das relações significativas na gravidez, em adolescentes e adultas, com um duplo foco - por um lado, nas características sociais e demográficas e nas condições anteriores de existência que estão associadas a um estilo de vinculação (in)seguro e, por outro lado, no impacto do estilo de vinculação na qualidade do relacionamento e do apoio por parte do companheiro e de uma outra pessoa significativa, na gravidez - com base na Attachment Style Interview - de Bifulco e colaboradores Os resultados revelam a importância de acontecimentos críticos do passado e do presente, tais como a separação ou divórcio parental durante a infância ou adolescência ou o desemprego no presente, associados a um estilo inseguro de vinculação. Além disso, os resultados apontam para o impacto do estilo de vinculação nas relações actuais, na medida em que os estilos inseguros estão associados a relacionamentos de menor qualidade na gravidez, com o companheiro e outros significativos.

$\mathrm{O}$ artigo de Matos e Costa inscreve-se na abordagem conceptual e metodológica de Bartholomew e pretende analisar a relação entre a vinculação aos pais e a vinculação ao par romântico e as diferenças de género do adolescente e da figura parental nas representações da vinculação, tendo como base duas entrevistas semi-estruturadas: Family Attachment Interview e Peer Attachment Interview de Bartholomew e Horowitz. As entrevistas dos adolescentes evidenciam a presença de regularidades nas representações de ambos os domínios, quer ao nível dos conteúdos, quer ao nível da qualidade da organização discursiva. Além desta proximidade na organização das narrativas, de um modo geral, tende também a haver proximidade na qualidade das representações da vinculação aos pais e ao par romântico: representações de vinculações seguras às figuras parentais tendem a estar associadas a representações de vinculações seguras no plano das relações românticas e a representações mais favoráveis acerca de si próprio e dos outros, não tendo sido encontradas diferenças do género do adolescente nos padrões de vinculação.

A pesquisa de João Moreira procura responder à questão "Será o estilo de vinculação específico para cada relação?", através de um estudo de generalizabilidade, com base num questionário medindo as duas dimensões fundamentais das auto-avaliações do estilo de vinculação dos adultos, a preocupação e a evitação, examinando em que grau os indivíduos exibem o mesmo estilo de vinculação em relações com diferentes parceiros. Os resultados deste estudo desafiam a noção da consistência em diferentes relações ao revelarem a necessidade de se considerar características específicas das relações, apoiando, assim, a proposta de Bartholomew (1990), de que a evitação reflecte a representação dos outros, sendo, por isso, específica das relações, enquanto que a preocupação tenderá a reflectir a representação de si próprio e, nesse sentido, será específica da pessoa. 
Os dois últimos artigos integrados neste número temático estão centrados na avaliação dos estilos de vinculação em adultos, com base em questionários de auto-relato. O artigo de Canavarro e colaboradores dá conta dos novos desenvolvimentos da Escala de Vinculação do Adulto (EVA), versão portuguesa da Adult Attachment Scale-R, de Collins e Read (1990). Tendo como ponto de partida os primeiros estudos de validação (Canavarro, 1999), a investigação actual integra uma amostra mais alargada da população clínica e não clínica, permitindo analisar a estrutura dimensional, fiabilidade e validade da escala. $\mathrm{O}$ estudo actual revela resultados favoráveis ao nível das características psicométricas da escala, a qual permite, agora, a classificação dos sujeitos com base nos protótipos identificados por Bartholomew e Horowitz (1991).

O estudo de Fonseca e colaboradores parte da versão portuguesa do Loving/Working: Are They Related?, de Hazan e Shaver (1990), para examinar o estilo de vinculação nos contextos das relações amorosas e das relações profissionais. Os resultados revelam que a maioria dos participantes se identifica com um estilo de vinculação segura e com uma orientação para o trabalho segura, tendo sido encontrada uma associação significativa entre estas duas variáveis. Por outro lado, um estilo de vinculação segura, nas relações amorosas e na orientação para o trabalho, parece estar associado a uma maior satisfação profissional e melhor adaptação ao trabalho.

Como procurámos evidenciar nesta apresentação, este número temático espelha a diversidade de temas e de métodos, dando voz à multiplicidade de focos sobre o território da investigação científica da vinculação, onde se cruzam várias abordagens a partir do legado Bowlby-Ainsworth-Main. Este cenário, marcado pela polifonia, constitui, também, um desafio à coerência teórica e à capacidade de dar sentido e de integrar os múltiplos dados e resultados, à luz do vasto património teórico e empírico. Esta diversidade traz novas questões, põe em cena velhas questões, coloca dúvidas onde estariam certezas. É por aqui que a ciência avança e é por aqui que a investigação da vinculação em Portugal nos parece estar, também, a caminhar.

\section{Referências}

Ainsworth, M., \& Wittig, B. (1969). Attachment and exploratory behavior of one-year-olds in a strange situation. In B. M. Foss (Ed.), Determinants of infant behavior (pp. 111-136). London: Methuen.

Ainsworth, M., \& Bell, S. (1970). Attachment, exploration, and separation: illustrated by the behavior of one-year-olds in a strange situation. Child Development, 41, 49-67. 
Ainsworth, M., Blehar, M., Waters, E., \& Wall, S. (1978). Patterns of attachment: a psychological study of the strange situation. Hillsdale: Erlbaum.

Bartholomew, K. (1990). Avoidance of intimacy: An attachment perspective. Journal of Social and Personal Relationships, 7, 147-178.

Bartholomew, K., \& Horowitz, L. (1991). Attachment styles among young adults: A test of a four category model. Journal of Personality and Social Psychology, 61 (2), 226-244.

Benoit, D., \& Parker, K. (1994). Stability and transmission of attachment among three generations. Infant Mental Health Journal, 10, 185-202.

Canavarro, M. C. (1999). Relações afectivas e saúde mental. Coimbra: Quarteto Editora.

Collins, N., \& Read, S. (1990). Adult attachment relationships, working models and relationship quality in dating couples. Journal of Personality and Social Psychology, 58, 644-683.

Fonagy, P., Steele, H., \& Steele, M. (1991). Maternal representations of attachment during pregnancy predict the organization of infant-mother attachment at one year of age. Child Development, 62, 891-905.

George, C., Kaplan, N., \& Main, M. (1984-1988). Adult Attachment Interview Protocol. Manuscrito não publicado, University of California at Berkeley.

Hazan, C., \& Shaver, P. (1990). Love and Work: An attachment Theoretical perspective. Journal of Personality and Social Psychology, 59, 270-280.

Soares, I., Lopes dos Santos, P., Jongenelen, I., Henriques, M. R., Silva, A. P., Figueiredo, B., Mascarenhas, C., Machado, G., Neves, L., Serra, M., Silva, M. C., Cunha, J. P., \& Costa, O. (1995). Avaliação da vinculação e da regulação autonómica da frequência cardíaca na situação estranha. Vol. IV. In L. S. Almeida, S. Araújo, M. M. Gonçalves, C. Machado, \& M. R. Simões (Eds.), Avaliação psicológica: formas e contextos (pp. 247-258). Braga: APPORT.

Soares, I. (1996). Representação da vinculação na idade adulta e na adolescência. Estudo intergeracional: mãe-filho(a). Braga: Centro de Estudos em Educação e Psicologia, Instituto de Educação e Psicologia, Universidade do Minho.

van IJzendoorn, M., \& Kroonenberg, P. (1988). Cross-cultural patterns of attachment: a meta-analysis of the strange situation. Child Development, 59, 147-156 .

van IJzendoorn, M., \& Bakermans-Kranenburg, M. (1996). Attachment represemtations in mothers, fathers, adolescents, and clinical groups: a meta-analytic search for normative data. Journal of Consulting and Clinical Psychology, 64, 8-21. 